\title{
Special issue on deep learning for emerging big multimedia super-resolution
}

\author{
Valerio Bellandi ${ }^{1} \cdot$ Abdellah Chehri $^{2} \cdot$ Salvatore Cuomo $^{3} \cdot$ Gwanggil Jeon $^{4}$ \\ Received: 11 May 2021 / Accepted: 19 May 2021 / Published online: 27 May 2021 \\ (c) The Author(s), under exclusive licence to Springer-Verlag GmbH Germany, part of Springer Nature 2021
}

\section{Introduction}

The main goal of the super-resolution (SR) is to restore a visually pleasing high-resolution image using a low-resolution image of video sequence. The higher resolution image is composed of higher pixel density with fine and precise details as compared with the low-resolution images or video. The majority of the applications, such as video surveillance, ultra-high-definition TV, low-resolution face recognition and remote sensing imaging are based on super-resolutions. Thus benefiting from the broader spectrun of these applications, the super-resolution has attracted massive interest form both acemedia and industry. And currently, a most active research field in today's era.

Previously, most of the reseachers focus on the machine learning techniques, such as supervised and unsupervised learning, reinforcement machine learning, naïve Bayes classifier, K-means clustering, random forests, and decision tree, etc. Using these techniques, the learning techniques are unable to provide fine and precise results. Therefore, due to the rapid advancements in the deep learning, deep networkbased high resolution has shown a promising performance in certain applications. Apperently, still many loops holes are still remaining that need serious attention. These loop-holes

Gwanggil Jeon

gjeon@inu.ac.kr

Valerio Bellandi

valerio.bellandi@unimi.it

Abdellah Chehri

Abdellah_Chehri@uqac.ca

Salvatore Cuomo

salvatore.cuomo@unina.it

Università degli Studi di Milano Statale, Milano, Italy

2 Université du Québec à Chicoutimi, Chicoutimi, Canada

3 University of Naples Federico II, Naples, Italy

4 Incheon National University, Incheon, Korea open new topics of deep learning for super-resolution images and videos, such as application includes, new objective functions, new architectures, large scale images, depth images, data acquisition, feature representation, time series analysis, knowledge understanding, and semantic modeling, various types of corruption, and new applications. There still exists a gap between extracting representations (or knowledge) from big multimedia data and practical demands.

\section{Themes of this special issue}

Starting from the above considerations, we solicited original contributions in four categories, all of which were expected to have an emphasis on deep learning and machine learning:

- State-of-the-art theories and novel application scenarios related to deep learning for SR for big multimedia data analytics.

- Novel time series analysis methods and applications.

- Surveys of recent progress in this area,

- The building of benchmark datasets.

This edition of the special issue serves as a forum for researchers all over the world to discuss their works and recent advances in deep learning for emerging big multimedia super-resolution. The special issue seeks for the original contribution of works that addresses the challenges of multimedia systems and is intended to provide a highly recognized international forum to present recent advances in multimedia systems. Papers addressing interesting realworld applications are especially encouraged. The list of possible topics includes, but not limited to:

- Supervised deep learning methods SR.

- Hybrid RGB and depth image SR with deep learning.

- Deep learning for large scale SR.

- Hardware and systems of deep learning for SR. 
- New image databases for deep learning for SR.

- Acceleration of deep learning for SR.

- Data Visualization patterns, query processing and analysis of big multimedia data.

- Business Intelligence for deep learning for SR.

- Deep learning framework for big multimedia data.

- Modern technologies for deep learning for SR.

- 2D/3D multimedia data understanding for deep learning for SR.

- Tools and applications for medicine and healthcare data (e.g. clustering, storing, ranking, hashing, and retrieval).

- Knowledge integration of multi-modal data through transfer learning and deep neural network.

After review, a total of 20 papers out of 30 submissions $(66.6 \%)$ have been accepted for publication in this issue.

\subsection{Models}

The contribution by Ullah et al. "Multi-feature-based crowd video modeling for visual event detection" proposes a novel method for modeling crowd video dynamics by adopting a two-stream convolutional architecture which incorporates spatial and temporal networks [1]. Their proposed method copes with the key challenge of capturing the complementary information on appearance from still frames and motion between frames. In the proposed method, a motion flow field is obtained from the video through dense optical flow. The authors demonstrate that the proposed method trained on multi-frame dense optical flow achieves significant improvement in performance in spite of limited training data. They train and evaluate the proposed method on a benchmark crowd video dataset. The experimental results of the method show that it outperforms five reference methods. The authors have chosen these reference methods since they are the most relevant to the work.

With time, AI technologies have matured well and resonated in various domains of applied sciences and engineering. The sub-domains of AI, machine learning (ML), deep learning (DL), and associated statistical tools are getting more attention. Therefore, various machine learning models are being created to take advantage of the data available and accomplish tasks, such as automatic prediction, classification, clustering, segmentation and anomaly detection, etc. Tasks like classification need labeled data used to train the models to achieve a reliable accuracy. The contribution by Bhatt et al. "The state of the art of deep learning models in medical science and their challenges" shows the systematic review of promising research areas and applications of DL models in medical diagnosis and medical healthcare systems [2]. The prevalent DL models, their architectures, and related pros, cons are discussed to clarify their prospects. Many deep learning networks have been useful in the field of medical image processing for prognosis and diagnosis of life-threatening ailments (e.g., breast cancer, lung cancer, and brain tumor, etc.), which stand as an error-prone and tedious task for doctors and specialists when performed manually. Medical images are processed using these DL methods to solve various tasks like prediction, segmentation, and classification with accuracy bypassing human abilities. However, the current DL models have some limitations that encourage the researchers to seek further improvement.

Image upsampling and noise removal are important tasks in digital image processing. Single-image upsampling and denoising influence the quality of the resulting images. Image upsampling is known as super-resolution (SR) and referred to as the restoration of a higher-resolution image from a given low-resolution image. In facial expression analysis, the resolution of the original image directly affects the reliability and validity of the emotional analysis. Hence, optimization of the resolution of the extracted original image during emotion analysis is important. In the contribution by Kim "Image super-resolution model using an improved deep learning-based facial expression analysis," a deep learning model is proposed, which is used on a single image [3]. The proposed super-resolution method is to classifies emotions from facial expressions.

Ulcerative colitis (UC) can be classified as proctitis, leftsided colitis or pancolitis, usually with rectal involvement at the beginning. Mucosal carcinogenesis is one of the most severe complications of UC. Persistent inflammation of the rectal mucosa may be an essential cause of mucosal cancer, thus the detection of rectal inflammation is of great significance. In the contribution by Zeng et al. "An image classification model based on transfer learning for ulcerative proctitis," the authors propose a transfer learning model to classify enteroscopy images to achieve adequate detection of ulcerative proctitis [4]. First, with the support of senior doctors, a dataset of ulcerative proctitis is created with 1450 endoscopic images. Then, with trained in the dataset, a new multi-model fusion network is proposed to classify ulcerative proctitis images. The proposed model combines three pre-trained models which are Xception, ResNet and DenseNet, and these pre-trained models are used to extract features from the images, then the extracted features are fed into a fully connected layer to predict the label of the input image. Experimental results show that, compared with other models, the proposed model has better performance, achieving the classification accuracy of $97.93 \%$, the sensitivity of $99 \%$ and the specificity of $99 \%$.

Recently, the introduction of Convolutional Neural Network (CNNs) has advanced the way of solving image segmentation tasks. Semantic image segmentation has considerably benefited from employing various CNN models. The most widely used network in this field is U-Net and its different variations. However, these models require a significant 
number of trainable parameters, floating-point operations per second, and great computational power to be trained. These factors make real-time semantic segmentation in lowpowered devices very hard. Therefore, in the present paper, the authors aim to modify particular aspects of the U-Net model to improve its performance through developing a fast U-Net (FU-Net) relying on bottleneck convolution layers in the contraction and expansion paths of the model. The contribution by Olimov et al. "FU-Net: fast biomedical image segmentation model based on bottleneck convolution layers" proposes a model which can be utilized in semantic segmentation applications even on the devices with limited computational power and memory by ensuring the state-of-the-art performance [5]. The amount of memory required by the proposed model is reduced by 23 times when compared with the original U-Net. Moreover, the modifications allowed achieving better performance. In conducted experiments, the authors assessed the performance of the proposed model on two biomedical image segmentation datasets, namely 2018 Data Science Bowl and ICIS 2018: Skin Lesion Analysis Towards Melanoma Detection. FU-Net demonstrated the state-of-the-art results in biomedical image segmentation, requiring the number of trainable parameters reduced by eight times compared with the original U-Net model. In addition, using bottleneck layers decreased the number of computations, resulting in nearly $30 \%$ speed-up at the training, validation and test stages. Furthermore, despite relying on fewer parameters FU-Net achieved a slight improvement of the performance in terms of pixel accuracy, Jaccard index, and dice coefficient evaluation metrics.

\subsection{Performance improvements}

Nowadays, highly sensitive medical images are vulnerable to data threats and privacy attacks. They must be kept secure while transmitting them across insecure channels precisely for this purpose. In the contribution by Sukumar et al. "Robust image steganography approach based on RIWTLaplacian pyramid and histogram shifting using deep learning," the authors focus on the robust image steganography by exploiting Redundant Integer Wavelet Transform (RIWT), Laplacian Pyramid, Arnold scrambling and Histogram shifting algorithm to facilitate secure communication of secret images in the context [6]. Stego images thus generated are subjected to a deep learning approach to assess if it can be classified as a cover or not. If not, the HS parameter is modified to generate stego images in such a way as to classify it as a cover image. Thus it is difficult to suspect the existence of a secret image by the Human Visual System (HVS). The efficiency of their method is analyzed by comparing it with related methods present in the literature. Average NCC values between the original secret image and the extracted secret image are 0.8917 which is higher than the schemes in the literature. Average PSNR values of the stego image are 36.375 even when the embedding rate is increased to 4 bits per pixel. The analysis was done on security and robustness also reveals better results. From the experimental analysis, it is proved that the proposed method is superior to the related methods of the literature.

Background subtraction, being the most cited algorithm for foreground detection, encounters the major problem of proper threshold value at run time. For the effective value of the threshold at run time in the background subtraction algorithm, the primary component of the foreground detection process, motion is used, in the proposed algorithm. For the said purpose, in the contribution by Khan et al. "Foreground detection using motion histogram threshold algorithm in high-resolution large datasets," the smooth histogram peaks and valley of the motion were analyzed, which reflects the high and slow motion areas of the moving object(s) in the given frame and generates the threshold value at run time by exploiting the values of peaks and valley [7]. This proposed algorithm was tested using four recommended video sequences, including indoor and outdoor shoots, and were compared with five high ranked algorithms. Based on the values of standard performance measures, the proposed algorithm achieved an average of more than $12.30 \%$ higher accuracy results.

Habitat mapping is an important and challenging task that helps in monitoring, managing, and preserving ecosystems. It becomes more challenging when marine habitats are mapped, as it is difficult to get quality images in an underwater environment. Moreover, achieving good location accuracy in underwater environments is an additional issue. Sonar imagery has good quality but is hard to be analyzed. Therefore, camera imagery is used for research purposes. The contribution by Yasir et al. "Habitat mapping using deep neural networks" targets marine habitats-more specifically, coral reef marine habitats [8]. Recognition of coral reef in underwater images poses a significant difficulty due to the nature of the data. Many species of coral reef have similar characteristics, i.e. higher inter-class similarity and lower intra-class similarity. Spatial borders between coral reef classes are hard to separate, as they tend to appear together in groups. For these reasons, the classification of coral reef species requires aid from marine biologists. This research work presents a technique for accurate coral reef classification using deep convolutional neural networks. The proposed approach has been validated on Moorea Labeled Corals (MLC), an imbalanced dataset, which is a subset of Moorea Coral Reef Long Term Ecological Research (MCR LTER) packaged for computer vision research. A custom valid patch dataset is extracted using the annotation files provided with the dataset. Two image enhancement algorithms and data-driven feature extraction techniques are employed using several pre-trained deep convolutional neural networks 
as feature extractors. Local-SPP technique is combined with feature extractors and followed by 2-layers multi-layer perceptron (MLP) classifier to achieve high classification rates.

One-dimensional encoding in optical communications has limitations in terms of the number of supported users and large bandwidth consumption. The contribution by Ahmed et al. "An efficient 2D encoding/decoding technique for optical communication system based on permutation vectors theory" presents a new algorithm to generate twodimensional (2D) encoding utilizing permutation vectors (PV) theory for incoherent multiple access network to suppress multiple access interference (MAI) and system complexity [9]. The proposed code design approach is based on spectral/spatial techniques for code generation. All possible combinations of PV code-sets are obtained by utilizing all permutations of the vectors with the repetition of each vector weight $(\mathrm{W})$ times. Furthermore, the 2D-PV code-set is constructed by combining two code sequences of the 1D-PV code. Furthermore, the transmitter-receiver architecture of the 2D-PV spectral/spatial (S/S) code -ased OCDMA system is presented. The Gaussian approximation is used to analyze the performance of the proposed OCDMA system with 2D- PV code by considering various noise sources (shot, thermal, and PIIN). Results indicate that the 2D-PV code provides increased cardinality by eliminating phase-induced intensity noise (PIIN) effects with a minimum likelihood of interference between multiple user data. Simulation implementations validate the proposed system performance for an agreeable bit error rate (BER) of $10^{-9}$.

Frequent itemset mining (FIM) is a significant data mining technique which is widely adopted in numerous applications for exploring frequent items. With the rapid growth and expansion of datasets, FIM has become an interesting topic for many researchers, which has triggered many innovations of numerous FIM algorithms in the big data environment. The contribution by Yimin et al. "PFIMD: a parallel MapReduce-based algorithm for frequent itemset mining" aims to design an optimization parallel frequent itemset mining algorithm based on MapReduce, named as PFIMD algorithm, to deal with the problem of time and space complexity during processing and computing item sets, as well as the failure to adequately balance the load among parallel tasks in the existing parallel FIM algorithms [10]. First, a structure called DiffNodeset is adopted for avoiding the increase of $\mathrm{N}$-list cardinality in the MRPrePost algorithm effectively. Then, a 2-way comparison strategy is designed to speed up the DiffNodeset generation of 2-itemsets and reduce the time complexity of the algorithm. Finally, the steps of the improved algorithm are parallelized using the cloud computing platform Hadoop and the programming model MapReduce. Moreover, to achieve a uniform grouping of each item in F-list, a load balancing strategy based on dynamic grouping is proposed, which solves the problem of an uneven load of each node in the cluster. The experimental results show that the modified algorithm not only overcomes the shortcoming of MRPrePost in the big data environment but also greatly reduces the time and space complexity. Finally, the specific applications of PFIMD algorithm in several multimedia data sets are listed to illustrate its universality.

With the rapid growth of big multimedia data, multimedia processing techniques are facing some challenges, such as knowledge understanding, semantic modeling, feature representation, etc. Hence, based on TextCNN and WGAN-gp (improved training of Wasserstein GANs), a deep learning framework is suggested to improve the efficiency of discriminating the specific style features and the style-independent content features in unpaired text style transfer for multimedia services. To redact a sentence with the requested style and preserve the style-independent content, the encoder-decoder framework is usually adopted. However, lacking of samecontent sentence pairs with different style for training, some works fail to capture the original content and generate satisfied style properties accurately in the transferred sentences. In the contribution by $\mathrm{Hu}$ et al. "A TextCNN and WGANgp-based deep learning frame for unpaired text style transfer in multimedia services," the authors adopt TextCNN to extract the style features in the transferred sentences and align the style features with the target style label by the generator (encoder and decoder) [11]. Meanwhile, WGAN-gp is utilized subtly to preserve the content features of original sentences. Experiments demonstrate that the performances of their framework on automatic evaluation and human evaluation are much better than the former works. Thus, it provides an effective method for unpaired text style transfer in multimedia services.

With the emergence of cyber technology, the biggest evolution has been observed in the use of Internet for financial purposes, in particular for the Internet banking sector. However, with the increase in the number of Internet banking users, many security issues have been discovered. In the recent past, there have been many successful cyber-attacks on Internet banking services (IBS) throughout the world. There exists a huge amount of various data about the security of the banking systems, however, proper analysis of such data using various learning techniques is needed for security assessment. In this research work, the authors aim to assess the security of IBS by developing a framework based on deep analysis of big data (available in various formats) and the existing security requirements of the country. In the contribution by Khattak et al. "An effective security assessment approach for Internet banking services via deep analysis of multimedia data," the authors propose a framework consisting of 93 data categories to assess the security of the IBS [12]. The authors evaluate their proposed approach on a case study consisting of the banks providing IBS in Pakistan. A total of 21 Pakistani banks providing Internet 
banking services are analyzed thoroughly using their proposed framework. The result uncovered many deficiencies in the Internet banking services of the analyzed banks. All these deficiencies are conveyed to the respective banks for verification and helping them to take corrective measures. In addition, a comprehensive set of security recommendations is developed for the banks, their customers and the regularity authority for improving Internet banking security.

Pupil detection in a human eye image or video plays a key role in many applications such as eye-tracking, diabetic retinopathy screening, smart homes, iris recognition, etc. Literature reveals pupil detection faces many complications including light reflections, cataract disease, pupil constriction/dilation moments, contact lenses, eyebrows, eyelashes, hair strips and closed eye. To cope with these challenges, research community has been struggling to devise resilient pupil localization schemes for the image/video data collected using the near infrared (NIR) or visible spectrum (VS) illumination. The contribution by Jan et al. "Pupil Detection Schemes in Human Eye: A Review" presents a critical review of numerous pupil detection schemes taken from standard sources [13]. This review includes pupil localization schemes based on machine learning, histogram/thresholding, Integro-differential operator (IDO), Hough transform and among others. Probable pros and cons of each scheme are highlighted. Finally, this study offers recommendations for designing a robust pupil detection system. As scope of pupil detection is very broader, therefore this review would be a great source of information for the relevant research community.

\subsection{Applications}

Skewness and obliqueness of vehicle plate images influence license plate recognition. The more tilted plate images are, the harder the recognition task is. To this end, if plate images are preprocessed to be aligned and rectified, the recognition performance would improve. In the contribution by Yoo and Jun "Deep corner prediction to rectify tilted license plate images," the authors propose deep neural network models that can locate four corner plate positions, which can then be used to perform the perspective transformation that can be used to rectify plates [14]. Such a transformation is called homography. The models consist of two sequential parts: a feature extraction part having convolution and a regression part with fully connected layers. The models are open in the sense that the feature extraction part can host other wellknown models such as Mobilenet as long as they have the feature capture capability. The authors devise a loss function as the sum of Euclidean distance between predicted coordinates and ground truth and discuss image augmentation schemes. The experiment results show that the models with well-known object detection models are able to predict corner positions with relatively high precision.

Managing the integrity of products and processes in a multi-stakeholder supply chain environment is a significant challenge. Many current solutions suffer from data fragmentation, lack of reliable provenance, and diverse protocol regulations across multiple distributions and processes. Amongst other solutions, Blockchain has emerged as a leading technology, since it provides secure traceability and control, immutability, and trust creation among stakeholders in a low-cost IT solution. Although Blockchain is making a significant impact in many areas, there are many impediments to its widespread adoption in supply chains. The contribution by Jabbar et al. "Blockchain-enabled supply chain: analysis, challenges, and future directions" is the first survey of its kind, with a detailed analysis of the challenges and future directions in Blockchain-enabled supply chains [15]. The authors review the existing digitalization of the supply chain including the role of GS1 standards and technologies. Current use cases and startups in the field of Blockchainenabled supply chains are reviewed and presented in tabulated form. Technical and non-technical challenges in the adoption of Blockchain for supply chain applications are critically analyzed, along with the suitability of various consensus algorithms for applications in the supply chain. The tools and technologies in the Blockchain ecosystem are depicted and analyzed. Some key areas as future research directions are also identified which must be addressed to realize mass adoption of Blockchain-based in supply chain traceability. Finally, the authors propose MOHBSChain, a novel framework for Blockchain-enabled supply chains.

Demosaicking is the way toward reproducing a full-hued picture from a deficient shaded picture. The single sensor doesn't catch all hues for a single pixel. To address this, a color filter array (CFA) is utilized to get a hued picture from a single sensor. The created picture from CFA is called a mosaic picture. In the contribution by Khadidos et al. "Bayer image demosaicking and denoising based on specialized networks using deep learning," the authors utilize specialized networks to remove the noise from Bayer images [16]. The mosaic picture is adulterated by commotion presented by a sensor or other equipment during catching. Demosaicking on the boisterous mosaic picture makes antiquities, for example, moiré and zippering. Some solutions have been proposed for denoising mosaic images but they are handcrafted solutions. In this paper, a solution is proposed to the first denoise and then demosaic the image using machine learning. The mosaic image is denoised using CNN which is then demosaicked using the residual learning strategy of a single specialized network. One of the networks is DHTN (deep high textured network) which is trained on textured images and the second one is DSTN (deep smooth textured network) which is trained on smooth images. Preliminary results show that 
the proposed approach generates better results and higher quality images than traditional approaches.

A skip-connection learning framework-based convolution neural network $(\mathrm{CNN})$ has recently achieved great success in image super-resolution (SR). However, most CNN models based on the skip-connection learning framework do not fully make use of potential multi-scale features of images. In the contribution by Liu et al. "Multi-scale skip-connection network for image super-resolution," the authors propose a multi-scale skip-connection network (MSN) to improve the visual quality of the image SR [17]. First, convolution kernels with different sizes are exploited to capture the multiscale features of LR images. All the feature-maps captured by convolution kernels of the same size are direct input into a multi-scale hybrid group (MHG); second, the convolution layers of each MHG are composed of dilated convolutions and standard convolutions. The hybrid convolutions can fully train feature details obtained from preceding and current scale convolution layers; three, the output of each hybrid convolution layer is fed into subsequent hybrid convolution layers by skip-connections, thus producing dense connections; lastly, the meta-upscale module is used as the upscale module, which can magnify the trained feature maps arbitrary scale factors. By being evaluated on a wide variety of images, the proposed MSN network achieves an advantage over the state-of-the-art methods in terms of both numerical results and visual quality.

With the improvement of SAR resolution, super-resolution SAR imaging is more and more widely used in all-time and all-weather video surveillance and remote sensing imaging. One implementation of super-resolution SAR is that radar works in the spotlight mode. In the case with highly squinted angle and acceleration, the azimuth space variance and the coupling between range and azimuth will become serious in super-resolution imaging. Thus, an azimuth frequency nonlinear chirp scaling algorithm is proposed to solve this problem. Based on the acceleration model, the accurate 2-D spectrum is performed by adopting the method of series reversion. The space-variance of missile-borne SAR in curved flight path is analyzed and an azimuth polynomial phase filter is constructed to make the coefficients of the perturbation function has sufficient flexibility to eliminate the spatial-variant couple terms between range and azimuth. In addition, the gradient operation is used to expand the space-variant coefficients of azimuth modulation term, and the perturbation function is applied to eliminate the space-variant in azimuth direction. The contribution by Qu et al. "A novel AFNCS algorithm for super-resolution SAR in curve trajectory" can process the missile-borne SAR data obtained in spotlight mode in highly squinted angle with high efficiency [18]. The simulation results verify the effectiveness of the proposed imaging approach. The integration of the research in this paper and the deep learning will further pave the way of super-resolution SAR imaging applications in disaster monitoring, security and surveillance.

With the development of deep learning (DL), convolutional neural networks (CNNs) have shown great reconstruction performance in single image super-resolution (SISR). However, some methods blindly deepen the networks to purchase the performance, which neglect to make full use of the multiscale information of different receptive fields and ignore the efficiency in practice. In the contribution by Zou et al. "LMSN:a lightweight multi-scale network for single image super-resolution," a lightweight SISR network with multi-scale information fusion blocks (MIFB) is proposed to fully extract information via a multiple ranges of receptive fields [19]. The features are refined in a coarse-to-fine manner within each block. Group convolutional layers are employed in each block to reduce the number of parameters and operations. Results of extensive experiments on the benchmarks show that their method achieves better performance than the state-of-the-arts with comparable parameters and multiply-accumulate (MAC) operations.

With the rapid development of intelligent transportation, the video surveillance system as its important component has been achieved much attention. Traffic condition closely related to people's lives needs to be tracked in time. Some methods estimate traffic flow by analyzing the pictures taken by fixed cameras. However, they can only estimate the traffic condition of particular roads. Different from the traditional traffic flow estimation methods, the contribution by Zhou et al. "A traffic flow estimation method based on unsupervised change detection" explores the video information rather than traffic images acquired by sensing remote-sensing sensors [20]. More specifically, the highlights of the work include the following parts: first, change detection is performed on analyzing the difference between one frame image extracted from Unmanned Aerial Vehicle (UAV) videos and an updated background image for the sake of recognizing the whole profile of every moving object. Second, a modified fuzzy c-means method is engaged in the process of change detection, which segments the road regions to enhance the profiles of moving objects and eliminate the noise of complex backgrounds. Finally, the estimation of traffic flow can be achieved by analyzing the change detection result. Besides, the videos shot by UAV on a crossroad are used to analyze the effectiveness of the proposed method. Experimental results on a series of binary images and proportion illustrations demonstrate the promising performance of the proposed method in terms of human visual perception and segmentation accuracy. 


\section{Conclusion}

The articles presented in this special issue provide insights in fields related to super-resolution in multimedia systems using artificial intelligence in deep learning algorithms, including models, performance evaluation and improvements, and application developments. We wish the readers can benefit from insights of these papers, and contribute to these rapidly growing areas. We also hope that this special issue would shed light on major developments in the area of Multimedia Systems and attract attention by the scientific community to pursue further investigations leading to the rapid implementation of these technologies.

Acknowledgements We would like to express our appreciation to all the authors for their informative contributions and the reviewers for their support and constructive critiques in making this special issue possible. Finally, we would like to express our sincere gratitude to Professor Changsheng $\mathrm{Xu}$, the Editor in Chief, for providing us with this unique opportunity to present our works in Springer Multimedia Systems.

\section{References}

1. Ullah, H., Islam, I.U., Ullah, M., et al.: Multi-feature-based crowd video modeling for visual event detection. Multimedia Syst (2020). https://doi.org/10.1007/s00530-020-00652-x

2. Bhatt, C., Kumar, I., Vijayakumar, V., et al.: The state of the art of deep learning models in medical science and their challenges. Multimedia Syst (2020). https://doi.org/10.1007/ s00530-020-00694-1

3. Kim, P.W.: Image super-resolution model using an improved deep learning-based facial expression analysis. Multimedia Syst (2020). https://doi.org/10.1007/s00530-020-00705-1

4. Zeng, F., Li, X., Deng, X., et al.: An image classification model based on transfer learning for ulcerative proctitis. Multimedia Syst (2021). https://doi.org/10.1007/s00530-020-00722-0

5. Olimov, B., Sanjar, K., Din, S., et al.: FU-Net: fast biomedical image segmentation model based on bottleneck convolution layers. Multimedia Syst (2021). https://doi.org/10.1007/ s00530-020-00726-w

6. Sukumar, A., Subramaniyaswamy, V., Ravi, L., et al.: Robust image steganography approach based on RIWT-Laplacian pyramid and histogram shifting using deep learning. Multimedia Syst (2020). https://doi.org/10.1007/s00530-020-00665-6

7. Khan, F.A., Nawaz, M., Imran, M., et al.: Foreground detection using motion histogram threshold algorithm in high-resolution large datasets. Multimedia Syst (2020). https://doi.org/10.1007/ s00530-020-00676-3

8. Yasir, M., Rahman, A.U., Gohar, M.: Habitat mapping using deep neural networks. Multimedia Syst (2020). https://doi.org/10.1007/ s00530-020-00695-0

9. Ahmed, H.Y., Zeghid, M., Imtiaz, W.A., et al.: An efficient 2D encoding/decoding technique for optical communication system based on permutation vectors theory. Multimedia Syst. (2020). https://doi.org/10.1007/s00530-020-00711-3

10. Yimin, M., Junhao, G., Mwakapesa, D.S., et al.: PFIMD: a parallel MapReduce-based algorithm for frequent itemset mining. Multimedia Syst (2021). https://doi.org/10.1007/s00530-020-00725-x

11. Hu, M., He, M., Su, W., et al.: A TextCNN and WGAN-gp based deep learning frame for unpaired text style transfer in multimedia services. Multimedia Syst (2020). https://doi.org/10.1007/ s00530-020-00714-0

12. Khattak, S., Jan, S., Ahmad, I., et al.: An effective security assessment approach for Internet banking services via deep analysis of multimedia data. Multimedia Syst (2020). https://doi.org/10.1007/ s00530-020-00680-7

13. Jan, F., et al.: Pupil detection schemes in human eye: a review. Multimedia Syst (2021). https://doi.org/10.1007/ s00530-021-00806-5

14. Yoo, H., Jun, K.: Deep corner prediction to rectify tilted license plate images. Multimedia Syst (2020). https://doi.org/10.1007/ s00530-020-00655-8

15. Jabbar, S., Lloyd, H., Hammoudeh, M., et al.: Blockchain-enabled supply chain: analysis, challenges, and future directions. Multimedia Syst (2020). https://doi.org/10.1007/s00530-020-00687-0

16. Khadidos, A.O., Khadidos, A.O., Khan, F.Q., et al.: Bayer image demosaicking and denoising based on specialized networks using deep learning. Multimedia Syst (2020). https://doi.org/10.1007/ s00530-020-00707-z

17. Liu, J., Ge, J., Xue, Y., et al.: Multi-scale skip-connection network for image super-resolution. Multimedia Syst (2020). https://doi. org/10.1007/s00530-020-00712-2

18. Qu, T., Zhang, Y., Wu, J.: A novel AFNCS algorithm for superresolution SAR in curve trajectory. Multimedia Syst (2020). https://doi.org/10.1007/s00530-020-00715-Z

19. Zou, Y., Yang, X., Albertini, M.K., et al.: LMSN:a lightweight multi-scale network for single image super-resolution. Multimedia Syst (2020). https://doi.org/10.1007/s00530-020-00720-2

20. Zhou, Y., Lei, Y., Yang, S., et al.: A traffic flow estimation method based on unsupervised change detection. Multimedia Syst (2021). https://doi.org/10.1007/s00530-020-00721-1

Publisher's Note Springer Nature remains neutral with regard to jurisdictional claims in published maps and institutional affiliations. 